\title{
Behavior Change Techniques and Delivery Modes in Interventions Targeting Adolescent Gambling: A Systematic Review
}

\author{
Tom St Quinton $^{1}$ (D) Ben Morris ${ }^{1} \cdot$ Dylan Pickering $^{2} \cdot$ Debbie M. Smith $^{3}$
}

Accepted: 29 January 2022 / Published online: 3 March 2022

(c) The Author(s), under exclusive licence to Springer Science+Business Media, LLC, part of Springer Nature 2022

\begin{abstract}
Background Adolescent gambling can lead to significant harms, yet participation rates continue to rise. Interventions targeting gambling reduction have been implemented in this population. However, it is not clear which behavior change techniques (BCTs) and modes of delivery (MOD) are most effective at reducing gambling.

Objective The objective of the study was to identify 'promising' BCTs and MODs by systematically reviewing interventions targeting adolescent gambling behavior. 'Promising' was defined as those present in at least $25 \%$ of all interventions and in at least two effective interventions.

Methods Three databases were searched (PsycINFO, Medline, and Scopus) from database inception to May 2021. Interventions were eligible if they were randomized controlled trials; targeting adolescents (aged 10-25 years); and assessing gambling behavior post-intervention. BCTs were identified using the Behavior Change Technique Taxonomy v1.

Results From the initial 3,315 studies, the removal of duplicates and ineligible articles resulted in sixteen studies included in the review. Eleven of these reported successfully reducing gambling behavior. Eighteen BCTs and six MODs were used across the interventions. The BCTs identified as promising were '4.2. Information about antecedents', '4.4. Behavioral experiments', '5.3. Information about social and environmental consequences', and '5.6. Information about emotional consequences'. Promising MODs were 'face-toface', 'computer', and 'playable electronic storage'.

Conclusions The study reviewed the content of interventions targeting adolescent gambling behavior. Four BCTs were identified as promising and should therefore be adopted in future interventions. To facilitate the delivery of these techniques, the study also identified three promising MODs. Interventions developed using these BCTs and MODs may successfully reduce adolescent gambling behavior.
\end{abstract}

Keywords Gambling $\cdot$ Behavior change techniques $\cdot$ Delivery mode $\cdot$ Adolescents

Extended author information available on the last page of the article 


\section{Introduction}

Gambling in adolescents has emerged as an increasing public health concern (Calado et al., 2017; Volberg et al., 2010). A systematic review undertaken by Calado et al. (2017) found that up to $12 \%$ of adolescents had gambling-related problems. Reported prevalence rates of problem gambling in adolescents are approximately 2-3 times higher than in adult populations (Shaffer \& Korn, 2002; Williams et al., 2012a); however, several researchers contend that certain situational and methodological issues have caused the rates to be overinflated (Delfabbro \& King, 2020). Irrespective of the accuracy of prevalence rates, problem gambling has many associated harms and specific to adolescents, research has shown that gambling can lead to financial issues, relationship problems, and poorer mental and physical health (Hardoon et al., 2004; Livazović \& Bojčić, 2019; Shaffer \& Hall, 2002). Adolescent gambling is also associated with the adoption of other detrimental health-related behaviors such as alcohol consumption (Svensson \& Sundqvist, 2019), substance abuse (Cook et al., 2015), risky driving (Proimos et al., 1998), and delinquent behaviors (Kryszajtys et al., 2018).

The accessibility of online gambling platforms has enabled adolescents to undertake the behavior more readily (Griffiths \& Parke, 2010). Internet gambling has demonstrated increasing popularity (Caillon et al., 2019), particularly in younger demographics (Hollén et al., 2020), with technology such as mobile apps facilitating this mode (Armitage, 2021). In addition to availability and convenience, adolescents are easily able to circumvent gambling age restrictions and bet anonymously (Canale et al., 2016; Delfabbro et al., 2009), or access illegal offshore betting sites (Messerlian et al., 2004). Gambling can begin as a form of entertainment but can quickly lead to significant problems in adolescents (Derevensky \& Gilbeau, 2015). Retrospective studies of clinical samples have shown that gambling onset typically occurs during the adolescent years and earlier onset is associated with greater problem severity (Burge et al., 2004). Similar findings were reported in a systematic review and meta-analysis of longitudinal studies that identified earlier gambling onset, number of gambling activities, and problem gambling severity as significant early risk factors for the subsequent development of gambling problems (Dowling et al., 2017). Moreover, despite age restrictions, the prevalence of gambling problems has been shown to be higher in adolescents than adults (Dowling et al., 2017; Nowak \& Aloe, 2014). It is therefore important that effective interventions exist to address and change gambling behaviors during adolescence (Oh et al., 2017).

\section{Behavior Change Techniques and Mode of Delivery}

Interventions promoting behavior change include strategies and methods to modify the behavior. Behavior change techniques (BCTs) are the “...observable, replicable, and irreducible component of an intervention designed to alter or redirect causal processes that regulate behaviour; that is, a technique is proposed to be an 'active ingredient'" (Michie et al., 2013, p. 82). They are the specific methods intervention designers employ to modify the behavior of interest. Michie et al. (2013) identified 93 unique BCTs in the BCT Taxonomy Version 1 (BCTTV1). Understanding the use of BCTs in intervention can help provide evidence of effectiveness. This can, in turn, inform the development of interventions in the future. For example, interventions demonstrating utility of a particular BCT would imply 
change efforts should also adopt the strategy whereas a BCT lacking in effectiveness would suggest developers refrain from its use. As an example, Michie et al. (2009) identified BCTs including 'Self-monitoring', 'Prompting intention formation', 'Goal setting', and 'Feedback' to be most effective in interventions promoting physical activity.

BCTs play an important role in behavior change, but intervention effectiveness is not only influenced by the content and strategies included. Another important component of behavior change interventions is the mode of delivery (MOD). The MOD concerns the way the intervention is delivered and the format features (Dombrowski et al., 2016; Marques et al., 2021). Thus, BCTs apply to what is delivered and the MOD relates to how this is achieved. Interventions can adopt a myriad of MODs such as face-to-face, online, telephone, or leaflets. Crucially, the effectiveness of an intervention can be influenced by the MOD (Marques et al., 2021). That is, whether behavior change is achieved can depend on how the intervention is communicated in practice. Therefore, it is important to examine both the content and delivery modes of interventions.

\section{Gambling Interventions}

Despite immense government and industry investment into gambling harm prevention, very few studies have systematically examined the BCTs and MODs of the numerous interventions developed to support this aim. Humphreys et al. (2021) recently identified the BCTs in web-based interventions targeting multiple health behaviors, including gambling. They found that effective interventions included '2.3. Self-monitoring of behavior', '2.2. Feedback on behavior', '6.2. Social comparison', and '4.1. Instruction on how to perform a behavior'. The authors did note, however, that only a limited number of strategies were included in interventions. In addition to this, Rodda et al. (2018) identified the BCTs included in therapist-delivered and self-help interventions for gambling problems. They found that some of the most frequently used strategies included '2.2. Feedback on behavior', '1.2. Problem solving', and '1.1. Goal setting (behavior)'.

Although these studies are useful in identifying intervention content and MODs, neither focused specifically on adolescents. Therefore, there exists a need to understand the components of interventions targeting adolescent gambling behavior. Moreover, the Humphreys et al. (2021) review was restricted to interventions conducted over the internet. As far as we are aware, no systematic review has identified the techniques and delivery modes adopted in such interventions. This work can appraise the state of current research and facilitate in the future development of effective interventions.

\section{Study Purpose}

The present review aimed to address the following questions:

1. What BCTs have been adopted in interventions targeting adolescent gambling behavior?

2. What BCTs have demonstrated the greatest effectiveness in interventions?

3. What modes of delivery have been adopted to deliver BCTs in interventions targeting adolescent gambling behavior?

4. What modes of delivery have demonstrated the greatest effectiveness in delivering BCTs in interventions? 


\section{Methods}

We followed the Preferred Reporting Items for Systematic Reviews and Meta-analyses (PRISMA) guidelines. The study was registered with PROSPERO, CRD42021254657.

\section{Eligibility Criteria}

Studies were included if they were randomized controlled trials of interventions targeting a reduction in adolescent (aged 10-25 years) gambling behavior. Gambling behavior was assessed using self-report or objective measures of gambling frequency or gambling expenditure. Studies assessing problem gambling were also included given the importance of this construct in the target literature. The intervention measured differences between the experimental condition and a control group. We excluded studies measuring only gambling cognitions such as urges or desires, unless accompanied by behavioral measures. Studies not published in English language and only including reviews, abstracts, opinion pieces, and letters to the editor were also excluded.

\section{Literature Search and Selection}

The following databases were used: PsycINFO, Medline, and Scopus. Searches were limited to articles published in peer reviewed journals between database inception and May 2021. Search terms included the following: (adolescents OR young adults* OR youth OR teenagers* OR students) AND (gambling* OR gamble) AND (intervention OR prevention OR program* OR treatment). Screened studies were imported into EndNote by one reviewer (TSQ). After the removal of duplicates, the lead author (TSQ) then screened the titles and abstracts of the articles identified through the searches. To check the reliability of the screening procedures, a second reviewer (BM) then screened $20 \%$ of these articles. Interrater reliability between the two reviewers was perfect $(\kappa=1.00)$. Full texts of potentially eligible studies were then screened by the lead author (TSQ), with a second reviewer (BM) again screening $20 \%$ of these articles to check reliability. At this stage, reliability was substantial with initial agreement on $83.3 \%$ of papers, $\kappa=0.75$. Differences were resolved through discussion. The reference lists of all identified articles were then hand-searched for further relevant studies.

\section{Data Extraction}

Two reviewers (TSQ \& BM) conducted data extraction using a purpose-designed data extraction sheet. This included the following: (1) General study information (author(s), date, country); (2) Aims; (3) Participants (sample size, age, and gender); (4) Measures (outcome, tool, follow-up period); (5) Intervention (conditions, MOD, provider, intensity, duration, and BCTs used); and (6) Findings.

Coding BCTs and MODs. The BCTTv1 was used to identify specific techniques included in intervention and control conditions. Following these principles, BCTs were extracted as "present beyond all reasonable doubt" (coded ++ ), or "present in all probability" (coded + ). To identify BCTs, we used descriptions provided in the paper and any additional materials made available. The main reviewer (TSQ) coded the techniques present in all studies and, 
to check reliability, a second reviewer (DMS) then coded $20 \%$ of these. The reliability of $\mathrm{BCT}$ coding was found to be high, $\kappa=0.84$. All discrepancies were then resolved through discussion. In terms of effectiveness, no gold standard approach exists for identifying BCTs (Michie et al., 2018). We therefore identified the 'promising' BCTs following a method adopted in previous studies (e.g., Ahmed et al., 2021; Brown et al., 2019, 2020; Lorencatto et al., 2012). Specifically, a BCT was defined as promising if it was present in at least $25 \%$ of all interventions and was present in at least two effective interventions. This approach can help identify the techniques with the greatest promise amongst those most frequently used (Brown et al., 2019). BCTs included in both the intervention and control condition were excluded. If multiple intervention conditions were included in a study, BCTs in effective arm(s) only were considered. We identified MODs using the ontology developed by Marques et al. (2021). Promising MODs were identified using the same process as the BCTs. That is, MODs included in $\geq 25 \%$ of all interventions and in at least two effective interventions were labeled 'promising'. There were no discrepancies between reviewers.

\section{Quality Assessment}

We assessed study quality using the revised Cochrane risk of bias tool for randomized trials (Sterne et al., 2019). We rated the risk of bias in five domains: (1) bias arising from the randomization process; (2) bias due to deviation from the intended interventions; (3) bias from missing outcome data; (4) bias in measurement of the outcome; (5) bias in selection of the reported results. We then classified each intervention as either: (1) low risk of bias, (2) some concerns, or (3) high risk of bias. Interventions were classified as having a low risk of bias when all domains were rated low risk; 'some concerns' of bias were indicated when at least one domain was assigned this rating; and high risk of bias was indicated when at least one domain was rated high risk. One reviewer (TSQ) conducted the assessment on all identified articles and a second reviewer (BM) checked $20 \%$ of articles. There were no discrepancies between reviewers.

\section{Results}

Figure 1 presents the flowchart of included papers. A total of 3,315 papers was identified through the search. After removing duplicates, the title and abstract of 2,142 papers were then screened, which led to the removal of 2,081 papers. The remaining 61 papers were then read in full. Full text screening led to 45 papers excluded for the following reasons: participants not meeting age criteria; no measure of behavior; not a randomized control trial; no statistical test performed; and duplicated data. No additional papers were identified in the hand-search, resulting in a total 16 papers included in the review (Broussard \& Wulfert, 2017; Calado et al., 2020; Canale et al., 2016; Donati et al., 2014, 2018, study 2; Gaboury \& Ladouceur 1993; Huic et al., 2017; Larimer et al., 2012; Martens et al., 2015; Petry et al., 2009; St-Pierre et al., 2017; Tani et al., 2021; Turner et al., 2008a, study 2; Turner et al., 2008b; Walther et al., 2013; Williams et al., 2010). 


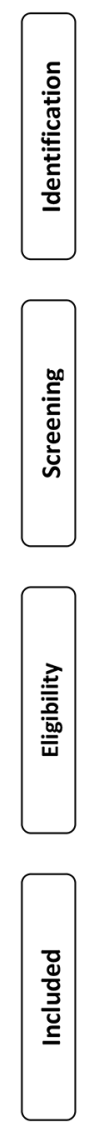

$$
\begin{aligned}
& \text { Records identified through database } \\
& \text { searching } \\
& (\mathrm{n}=3,315)
\end{aligned}
$$

Additional records identified through other sources $(\mathrm{n}=0)$

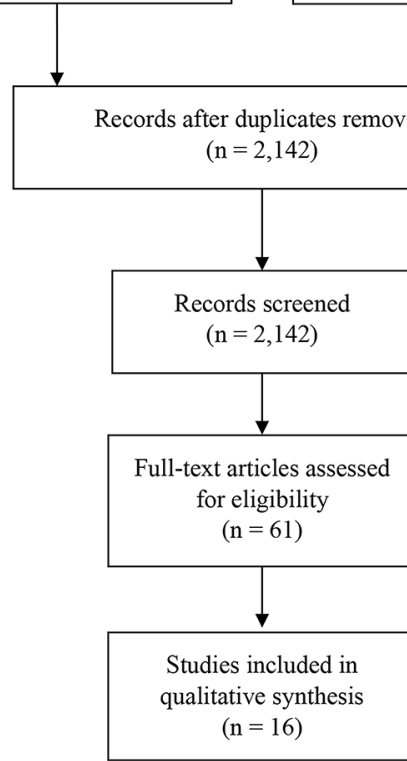

Records excluded $(\mathrm{n}=2,081)$

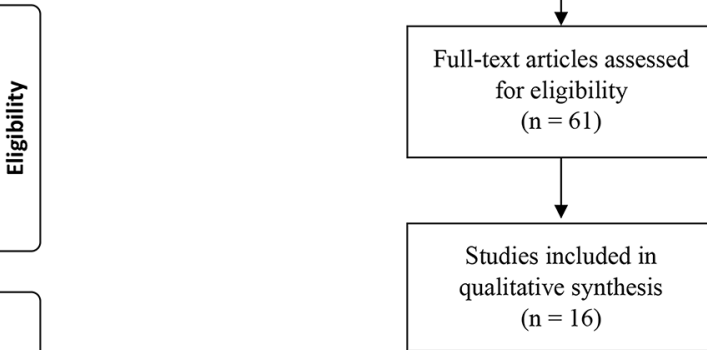

Full-text articles excluded, with reasons $(\mathrm{n}=45)$

- Not an RCT $(n=10)$

- No measure of behavior $(\mathrm{n}=11)$

- Not adolescents $(\mathrm{n}=22)$

- No statistical test $(n=1)$

- Uses the same data $(n=$ 1)

Fig. 1 Flow diagram

\section{Study Characteristics and Quality Assessment}

Table 1 shows the characteristics of the eligible studies. Studies were conducted most frequently in Canada (Gaboury \& Ladouceur 1993; St-Pierre et al., 2017; Turner et al., 2008a, study 2; Turner et al., 2008b; Williams et al., 2010), with four undertaken in the USA (Broussard \& Wulfert; Larimer et al., 2012; Martens et al., 2015; Petry et al., 2009), four in Italy Canale et al., 2016; Donati et al., 2014, 2018, study 2; Tani et al., 2021), and one in Croatia (Huic et al., 2017), Portugal (Calado et al., 2020), and Germany (Walther et al., 2013). A total of 6,703 participants were included in the studies $(\min =34 ; \max =2,109)$. The interventions were delivered by researchers, psychologists, therapists, students, research assistants, and teachers. Where reported, intervention duration ranged from 1 to 7 weeks, with the number of sessions also varying from 1 to 7 . The follow-up assessment period ranged from imme- 
Table 1 Study characteristics

\begin{tabular}{|c|c|c|c|c|c|}
\hline $\begin{array}{l}\text { Gen- } \\
\text { eral study } \\
\text { information }\end{array}$ & Aims & Participants & Measures & Intervention & Findings \\
\hline $\begin{array}{l}\text { Broussard } \\
\text { \& Wulfert } \\
(2017) . \\
\text { USA }\end{array}$ & $\begin{array}{l}\text { Tested the ef- } \\
\text { fects of a digital } \\
\text { slot machine } \\
\text { intervention } \\
\text { on a gambling } \\
\text { analogue task }\end{array}$ & $\begin{array}{l}90 \text { college } \\
\text { students } \\
\text { Age: } \\
\text { mean }=19.6 \\
\text { Gender: } \\
\text { males }=45 ; \\
\text { females }=45\end{array}$ & $\begin{array}{l}\text { Outcome }(s) \text { : } \\
\text { gambling } \\
\text { frequency } \\
\text { Tool(s): slot } \\
\text { machine } \\
\text { Follow-up: } \\
\text { immediately } \\
\text { post-intervention }\end{array}$ & $\begin{array}{l}\text { Delivery mode: video } \\
\text { game } \\
\text { Provider: researcher } \\
\text { Intensity and dura- } \\
\text { tion: } 1 \times 10 \text { min } \\
\text { BCTs used: } 4.4 \text {. Be- } \\
\text { havioral experiments } \\
\text { Control group } \\
\text { received a handout } \\
\text { unrelated to gambling }\end{array}$ & $\begin{array}{l}\text { Inter- } \\
\text { vention } \\
\text { condition } \\
\text { played sig- } \\
\text { nificantly } \\
\text { fewer trials } \\
\text { than the } \\
\text { control }\end{array}$ \\
\hline $\begin{array}{l}\text { Calado et al. } \\
(2020) . \\
\text { Portugal }\end{array}$ & $\begin{array}{l}\text { Evaluated a } \\
\text { youth gambling } \\
\text { prevention } \\
\text { program }\end{array}$ & $\begin{array}{l}111 \text { students } \\
\text { Age: } \\
\text { mean }=17.6 \\
\text { Gender: } \\
\text { males }=46, \\
\text { females }=65\end{array}$ & $\begin{array}{l}\text { Outcome }(s) \text { : } \\
\text { gambling } \\
\text { frequency; } \\
\text { gambling } \\
\text { expenditure } \\
\text { Tool }(s) \text { : } \\
\text { researcher-gen- } \\
\text { erated questions; } \\
\text { DSM-IV-MR-J } \\
\text { Follow-up: } \\
\text { immediately } \\
\text { and 6-weeks } \\
\text { post-intervention }\end{array}$ & $\begin{array}{l}\text { Delivery mode: face- } \\
\text { to face } \\
\text { Provider: researcher } \\
\text { Intensity and dura- } \\
\text { tion: } 5 \times 1 \text {-hour, once } \\
\text { per week } \\
\text { BCTs used: } 3.1 \text {. Social } \\
\text { support (unspeci- } \\
\text { fied); } 4.4 \text {. Behavioral } \\
\text { experiments; } 5.3 \text {. } \\
\text { Information about } \\
\text { social and environ- } \\
\text { mental consequences; } \\
\text { 5.6. Information } \\
\text { about emotional } \\
\text { consequences } \\
\text { Control group com- } \\
\text { pleted assessments } \\
\text { only }\end{array}$ & $\begin{array}{l}\text { Gambling } \\
\text { frequency } \\
\text { sig- } \\
\text { nificantly } \\
\text { decreased } \\
\text { in inter- } \\
\text { vention } \\
\text { condition } \\
\text { immedi- } \\
\text { ately after } \\
\text { the inter- } \\
\text { vention } \\
\text { and these } \\
\text { effects } \\
\text { were main- } \\
\text { tained at } \\
\text { the 6-week } \\
\text { follow- } \\
\text { up. No } \\
\text { change in } \\
\text { control. No } \\
\text { change in } \\
\text { gambling } \\
\text { expenditure } \\
\text { in interven- } \\
\text { tion and } \\
\text { control }\end{array}$ \\
\hline
\end{tabular}


Table 1 (continued)

\begin{tabular}{|c|c|c|c|c|c|}
\hline $\begin{array}{l}\text { Gen- } \\
\text { eral study } \\
\text { information }\end{array}$ & Aims & Participants & Measures & Intervention & Findings \\
\hline $\begin{array}{l}\text { Canale et al. } \\
(2016) . \\
\text { Italy }\end{array}$ & $\begin{array}{l}\text { Tested a web- } \\
\text { based gambling } \\
\text { intervention } \\
\text { targeting high- } \\
\text { school students }\end{array}$ & $\begin{array}{l}168 \text { students } \\
\text { Age: } \\
\text { mean }=15 ; \\
\text { range }=14-18 \\
\text { Gender: } \\
\text { male }=58 \%\end{array}$ & $\begin{array}{l}\text { Outcome }(s) \text { : } \\
\text { gambling } \\
\text { frequency; } \\
\text { gambling expen- } \\
\text { diture; gambling } \\
\text { problems } \\
\text { Tool(s): SOGS- } \\
\text { RA; researcher- } \\
\text { generated } \\
\text { questions } \\
\text { Follow-up: } \\
2 \text { months } \\
\text { post-intervention }\end{array}$ & $\begin{array}{l}\text { Delivery mode: } \\
\text { website } \\
\text { Provider: researcher } \\
\text { Intensity and dura- } \\
\text { tion: } 3 \text { weeks } \\
\text { BCTs used: } 2.2 \text {. Feed- } \\
\text { back on behavior; } 4.1 \text {. } \\
\text { Instruction on how to } \\
\text { perform the behavior; } \\
4.2 \text {. Information about } \\
\text { antecedents; } 5.3 \text {. } \\
\text { Information about } \\
\text { social and environ- } \\
\text { mental consequences; } \\
\text { 5.6. Information } \\
\text { about emotional } \\
\text { consequences; } 14.2 \text {. } \\
\text { Punishment } \\
\text { Control group } \\
\text { received personalized } \\
\text { feedback only }\end{array}$ & $\begin{array}{l}\text { Inter- } \\
\text { vention } \\
\text { condition } \\
\text { reduced } \\
\text { gambling } \\
\text { problems } \\
\text { compared } \\
\text { to the } \\
\text { control. No } \\
\text { differences } \\
\text { in gam- } \\
\text { bling fre- } \\
\text { quency and } \\
\text { expenditure }\end{array}$ \\
\hline $\begin{array}{l}\text { Donati et al. } \\
(2018 \text {, study } \\
2) . \\
\text { Italy }\end{array}$ & $\begin{array}{l}\text { Tested a school- } \\
\text { based interven- } \\
\text { tion targeting } \\
\text { gambling-related } \\
\text { cognitive distor- } \\
\text { tions and gam- } \\
\text { bling frequency }\end{array}$ & $\begin{array}{l}34 \text { high school } \\
\text { students } \\
\text { Age: } \\
\text { mean }=16.8 ; \\
\text { range: } 15-19 \\
\text { Gender: all } \\
\text { male }\end{array}$ & $\begin{array}{l}\text { Outcome }(s) \text { : } \\
\text { gambling } \\
\text { frequency } \\
\text { Tool }(s) \text { : } \\
\text { SOGS-RA } \\
\text { Follow-up: } \\
\text { immediately } \\
\text { and } 6 \text { months } \\
\text { post-intervention }\end{array}$ & $\begin{array}{l}\text { Delivery mode: face- } \\
\text { to face } \\
\text { Provider: develop- } \\
\text { mental psychologist } \\
\text { and two operators } \\
\text { from an addiction unit } \\
\text { Intensity and dura- } \\
\text { tion: } 2 \times 2 \text {-hour, once } \\
\text { per week } \\
\text { BCTs used: } 4.2 \text {. Infor- } \\
\text { mation about anteced- } \\
\text { ents; } 4.4 \text {. Behavioral } \\
\text { experiments } \\
\text { Control group com- } \\
\text { pleted assessments } \\
\text { only }\end{array}$ & $\begin{array}{l}\text { Significant } \\
\text { reduc- } \\
\text { tion in } \\
\text { gambling } \\
\text { frequency } \\
\text { in interven- } \\
\text { tion condi- } \\
\text { tion but no } \\
\text { change in } \\
\text { control }\end{array}$ \\
\hline
\end{tabular}


Table 1 (continued)

\begin{tabular}{|c|c|c|c|c|c|}
\hline $\begin{array}{l}\text { Gen- } \\
\text { eral study } \\
\text { information }\end{array}$ & Aims & Participants & Measures & Intervention & Findings \\
\hline $\begin{array}{l}\text { Donati et al. } \\
(2014) . \\
\text { Italy }\end{array}$ & $\begin{array}{l}\text { Tested the ef- } \\
\text { fectiveness of } \\
\text { an integrative } \\
\text { gambling inter- } \\
\text { vention targeting } \\
\text { adolescent prob- } \\
\text { lem gambling }\end{array}$ & $\begin{array}{l}181 \\
\text { adolescents } \\
\text { Age: } \\
\text { mean }=15.9 ; \\
\text { range }=15-18 \\
\text { Gender: } \\
\text { male }=64 \%\end{array}$ & $\begin{array}{l}\text { Outcome }(s) \text { : } \\
\text { problem } \\
\text { gambling } \\
\text { Tool }(s) \text { : } \\
\text { SOGS-RA } \\
\text { Follow-up: } \\
\text { immediately } \\
\text { and 6-months } \\
\text { post-intervention }\end{array}$ & $\begin{array}{l}\text { Delivery mode: face- } \\
\text { to face; computer; } \\
\text { playable electronic } \\
\text { storage } \\
\text { Provider: develop- } \\
\text { mental psychologist } \\
\text { Intensity and dura- } \\
\text { tion: } 2 \times 2 \text {-hour, once } \\
\text { per week } \\
\text { BCTs used: } 4.2 \text {. } \\
\text { Information about } \\
\text { antecedents; } 4.4 \text {. Be- } \\
\text { havioral experiments; } \\
\text { 5.3. Information about } \\
\text { social and environ- } \\
\text { mental consequences } \\
\text { Control group com- } \\
\text { pleted assessments } \\
\text { only }\end{array}$ & $\begin{array}{l}\text { Significant } \\
\text { reduc- } \\
\text { tion in the } \\
\text { percentage } \\
\text { of gam- } \\
\text { blers and } \\
\text { problem } \\
\text { gamblers } \\
\text { in the in- } \\
\text { tervention } \\
\text { condition; } \\
\text { how- } \\
\text { ever, no } \\
\text { comparison } \\
\text { with con- } \\
\text { trol group } \\
\text { reported }\end{array}$ \\
\hline $\begin{array}{l}\text { Gaboury \& } \\
\text { Ladouceur. } \\
\text { (1993). } \\
\text { Canada }\end{array}$ & $\begin{array}{l}\text { Evaluated a } \\
\text { gambling pre- } \\
\text { vention program }\end{array}$ & $\begin{array}{l}289 \text { high } \\
\text { school } \\
\text { students } \\
\text { Age: } \\
\text { mean=16 } \\
\text { Gender: ns }\end{array}$ & $\begin{array}{l}\text { Outcome }(s) \text { : } \\
\text { gambling } \\
\text { frequency; } \\
\text { gambling } \\
\text { expenditure } \\
\text { Tool }(s) \text { : research- } \\
\text { er-generated } \\
\text { questions } \\
\text { Follow-up: } \\
\text { immediately } \\
\text { and 6-months } \\
\text { post-intervention }\end{array}$ & $\begin{array}{l}\text { Delivery mode: } \\
\text { face-to face; playable } \\
\text { electronic storage } \\
\text { Provider: research } \\
\text { assistants } \\
\text { Intensity and dura- } \\
\text { tion: } 3 \times 75 \text { min, over } \\
3 \text { weeks } \\
\text { BCTs used: } 5.1 \text {. Infor- } \\
\text { mation about health } \\
\text { consequences; } 5.3 \text {. } \\
\text { Information about } \\
\text { social and environ- } \\
\text { mental consequences; } \\
6.1 . \text { Demonstration } \\
\text { of the behavior; } 9.1 \text {. } \\
\text { Credible source; } \\
16.3 \text {. Vicarious } \\
\text { consequences } \\
\text { Control group com- } \\
\text { pleted assessments } \\
\text { only }\end{array}$ & $\begin{array}{l}\text { No } \\
\text { significant } \\
\text { differences }\end{array}$ \\
\hline
\end{tabular}


Table 1 (continued)

\begin{tabular}{|c|c|c|c|c|c|}
\hline $\begin{array}{l}\text { Gen- } \\
\text { eral study } \\
\text { information }\end{array}$ & Aims & Participants & Measures & Intervention & Findings \\
\hline $\begin{array}{l}\text { Huic et al. } \\
\text { (2017). } \\
\text { Croatia }\end{array}$ & $\begin{array}{l}\text { Pilot evaluation } \\
\text { of a school- } \\
\text { based gambling } \\
\text { prevention } \\
\text { program }\end{array}$ & $\begin{array}{l}190 \text { high } \\
\text { school } \\
\text { students } \\
\text { Age: } \\
\text { mean }=15.6 ; \\
\text { range }=14-17 \\
\text { Gender: } \\
\text { male }=67.6 \%\end{array}$ & $\begin{array}{l}\text { Outcome }(s) \text { : } \\
\text { gambling } \\
\text { frequency; gam- } \\
\text { bling problems } \\
\text { Tool(s): research- } \\
\text { er generated } \\
\text { questions; CAGI } \\
\text { Follow-up: } \\
\text { immediately } \\
\text { post-intervention }\end{array}$ & $\begin{array}{l}\text { Delivery mode: face- } \\
\text { to face } \\
\text { Provider: two trainers } \\
\text { Intensity and dura- } \\
\text { tion: } 6 \times 90 \text { min, } \\
\text { weeks ns } \\
\text { BCTs used: } 1.2 \text {. } \\
\text { Problem solving; } 5.1 \text {. } \\
\text { Information about } \\
\text { health consequences; } \\
\text { 5.3. Information about } \\
\text { social and environ- } \\
\text { mental consequences; } \\
\text { 5.6. Information } \\
\text { about emotional } \\
\text { consequences; } 8.1 \text {. } \\
\text { Behavioral practice/ } \\
\text { rehearsal } \\
\text { Control group had } \\
\text { regular school } \\
\text { activities }\end{array}$ & $\begin{array}{l}\text { No } \\
\text { significant } \\
\text { differences }\end{array}$ \\
\hline
\end{tabular}


Table 1 (continued)

\begin{tabular}{|c|c|c|c|c|c|}
\hline $\begin{array}{l}\text { Gen- } \\
\text { eral study } \\
\text { information }\end{array}$ & Aims & Participants & Measures & Intervention & Findings \\
\hline $\begin{array}{l}\text { Larimer et al. } \\
(2012) \text {. } \\
\text { USA }\end{array}$ & $\begin{array}{l}\text { Evaluated an } \\
\text { intervention tar- } \\
\text { geting gambling } \\
\text { in at-risk college } \\
\text { students }\end{array}$ & $\begin{array}{l}147 \text { college } \\
\text { students } \\
\text { Age: } \\
\text { mean }=21.2 ; \\
\text { range }=19-25 \\
\text { Gender: } \\
\text { male }=65.3 \%\end{array}$ & $\begin{array}{l}\text { Outcome }(s) \text { : } \\
\text { gambling } \\
\text { frequency; } \\
\text { gambling expen- } \\
\text { diture; gambling } \\
\text { problems } \\
\text { Tool(s): GQPN; } \\
\text { GPI } \\
\text { Follow-up: } \\
6 \text { months } \\
\text { post-intervention }\end{array}$ & $\begin{array}{l}\text { Two intervention con- } \\
\text { ditions: PFI and CBI } \\
\text { Delivery mode: } \\
\text { face-to face; printed } \\
\text { publication } \\
\text { Provider: trained } \\
\text { therapists (graduate } \\
\text { students) } \\
\text { Intensity and } \\
\text { duration: PFI: } \\
1 \times 60-90 \text { min; CBI: } \\
4-6 \text { hourly sessions, } \\
\text { once per week } \\
\text { BCTs used: } 1.2 \text {. } \\
\text { Problem solving; } 2.2 \text {. } \\
\text { Feedback on behav- } \\
\text { ior; } 2.3 \text {. Self-moni- } \\
\text { toring of behavior; } \\
3.1 . \text { Social support } \\
\text { (unspecified); } 4.1 \text {. } \\
\text { Instruction on how to } \\
\text { perform the behavior; } \\
4.2 . \text { Information about } \\
\text { antecedents; } 5.3 \text {. } \\
\text { Information about } \\
\text { social and environ- } \\
\text { mental consequences; } \\
5.6 \text {. Information } \\
\text { about emotional con- } \\
\text { sequences; } 6.2 . \text { Social } \\
\text { comparison; } 8.1 . \\
\text { Behavioral practice/ } \\
\text { rehearsal } \\
\text { Control group com- } \\
\text { pleted assessments } \\
\text { only }\end{array}$ & $\begin{array}{l}\text { PFI } \\
\text { condition } \\
\text { reduced } \\
\text { gambling } \\
\text { frequency } \\
\text { and } \\
\text { gambling } \\
\text { problems } \\
\text { compared } \\
\text { to control. } \\
\text { No differ- } \\
\text { ences in } \\
\text { gambling } \\
\text { expendi- } \\
\text { ture. No } \\
\text { differences } \\
\text { in CBI } \\
\text { condition }\end{array}$ \\
\hline
\end{tabular}


Table 1 (continued)

\begin{tabular}{|c|c|c|c|c|c|}
\hline $\begin{array}{l}\text { Gen- } \\
\text { eral study } \\
\text { information }\end{array}$ & Aims & Participants & Measures & Intervention & Findings \\
\hline $\begin{array}{l}\text { Martens et al. } \\
(2015) \text {. } \\
\text { USA }\end{array}$ & $\begin{array}{l}\text { Tested an inter- } \\
\text { vention targeting } \\
\text { gambling be- } \\
\text { havior in at-risk } \\
\text { college students }\end{array}$ & $\begin{array}{l}333 \text { students } \\
\text { Age: } \\
\text { mean }=21.9 \\
\text { Gender: } \\
\text { male }=60 \% ; \\
\text { female }=40 \%\end{array}$ & $\begin{array}{l}\text { Outcome(s): } \\
\text { gambling } \\
\text { frequency; } \\
\text { gambling expen- } \\
\text { diture; gambling } \\
\text { problems } \\
\text { Tool: G-TLFB; } \\
\text { CAGI } \\
\text { Follow-up: } \\
3 \text { months } \\
\text { post-intervention }\end{array}$ & $\begin{array}{l}\text { Two intervention } \\
\text { conditions: PFI and } \\
\text { EDU } \\
\text { Delivery mode: } \\
\text { face-to face; printed } \\
\text { publication } \\
\text { Provider: researcher } \\
\text { Intensity and dura- } \\
\text { tion: } 1 \times 10 \text { min } \\
\text { BCTs used: } 2.2 \text {. Feed- } \\
\text { back on behavior; } 4.1 \text {. } \\
\text { Instruction on how to } \\
\text { perform the behavior; } \\
4.2 \text {. Information about } \\
\text { antecedents; } 6.2 \text {. } \\
\text { Social comparison } \\
\text { Control group com- } \\
\text { pleted assessments } \\
\text { only }\end{array}$ & $\begin{array}{l}\text { PFI } \\
\text { condition } \\
\text { gambled } \\
\text { less money } \\
\text { and report- } \\
\text { ed fewer } \\
\text { gambling- } \\
\text { related } \\
\text { problems } \\
\text { than the } \\
\text { control. No } \\
\text { difference } \\
\text { in fre- } \\
\text { quency. No } \\
\text { differences } \\
\text { between } \\
\text { the EDU } \\
\text { and con- } \\
\text { trol, or the } \\
\text { PFI and } \\
\text { the EDU } \\
\text { conditions }\end{array}$ \\
\hline $\begin{array}{l}\text { Petry et al. } \\
\text { (2009). } \\
\text { USA }\end{array}$ & $\begin{array}{l}\text { Tested brief } \\
\text { interventions tar- } \\
\text { geting gambling } \\
\text { behavior in col- } \\
\text { lege students }\end{array}$ & $\begin{array}{l}117 \text { students } \\
\text { Age: } \\
\text { mean }=20.3 \\
\text { Gender: } \\
\text { male }=99\end{array}$ & $\begin{array}{l}\text { Outcome }(s) \text { : } \\
\text { gambling } \\
\text { frequency; } \\
\text { gambling expen- } \\
\text { diture; gambling } \\
\text { problems } \\
\text { Tool(s): ASI-G; } \\
\text { researcher-gener- } \\
\text { ated questions } \\
\text { Follow-up: } \\
6 \text { weeks and } \\
9 \text { months } \\
\text { post-intervention }\end{array}$ & $\begin{array}{l}\text { Three intervention } \\
\text { conditions: Brief ad- } \\
\text { vice, MET, and MET } \\
+ \text { CBT } \\
\text { Delivery mode: } \\
\text { face-to face; printed } \\
\text { publication } \\
\text { Provider: trained } \\
\text { therapists } \\
\text { Intensity and dura- } \\
\text { tion: Brief advice: } \\
1 \times 10-15 \text { min; MET: } \\
1 \times 50 \text { min; MET + } \\
\text { CBT: } 1 \times 50 \text { min for } \\
\text { MET \& } 3 \text { weekly for } \\
\text { CBT } \\
\text { BCTs used: } 1.2 \text {. } \\
\text { Problem solving; } 2.2 \text {. } \\
\text { Feedback on behav- } \\
\text { ior; } 4.2 . \text { Information } \\
\text { about antecedents; } \\
6.2 \text {. Social com- } \\
\text { parison; } 9.2 . \text { Pros and } \\
\text { cons } \\
\text { Control group com- } \\
\text { pleted assessments } \\
\text { only }\end{array}$ & $\begin{array}{l}\text { Significant } \\
\text { decrease in } \\
\text { gambling } \\
\text { frequency, } \\
\text { expendi- } \\
\text { ture, and } \\
\text { problems } \\
\text { in all in- } \\
\text { tervention } \\
\text { conditions } \\
\text { compared } \\
\text { to control. } \\
\text { However, } \\
\text { gambling } \\
\text { problems } \\
\text { and expen- } \\
\text { diture sig- } \\
\text { nificantly } \\
\text { decreased } \\
\text { in the MET } \\
\text { group only } \\
\text { compared } \\
\text { to the con- } \\
\text { trol after } 9 \\
\text { months }\end{array}$ \\
\hline
\end{tabular}


Table 1 (continued)

\begin{tabular}{|c|c|c|c|c|c|}
\hline $\begin{array}{l}\text { Gen- } \\
\text { eral study } \\
\text { information }\end{array}$ & Aims & Participants & Measures & Intervention & Findings \\
\hline $\begin{array}{l}\text { St-Pierre } \\
\text { et al. } \\
(2017) \text {. } \\
\text { Canada }\end{array}$ & $\begin{array}{l}\text { Evaluated a } \\
\text { school-based } \\
\text { gambling pre- } \\
\text { vention program }\end{array}$ & $\begin{array}{l}280 \text { high } \\
\text { school } \\
\text { students } \\
\text { Age: } \\
\text { mean }=15.1 ; \\
\text { range }=13-17 \\
\text { Gender: } \\
\text { male }=140\end{array}$ & $\begin{array}{l}\text { Outcome }(s) \text { : } \\
\text { gambling } \\
\text { frequency } \\
\text { Tool(s): GAQ } \\
\text { Follow-up: } \\
3 \text { months } \\
\text { post-intervention }\end{array}$ & $\begin{array}{l}\text { Delivery mode: } \\
\text { face-to face; playable } \\
\text { electronic storage } \\
\text { Provider: program fa- } \\
\text { cilitators and research } \\
\text { assistants } \\
\text { Intensity and dura- } \\
\text { tion: } 2 \times 25 \text {-min, once } \\
\text { per week } \\
\text { BCTs used: } 5.1 \text {. Infor- } \\
\text { mation about health } \\
\text { consequences; } 5.3 \text {. } \\
\text { Information about } \\
\text { social and environ- } \\
\text { mental consequences; } \\
\text { 5.6. Information } \\
\text { about emotional } \\
\text { consequences; } 6.3 \text {. } \\
\text { Information about } \\
\text { others' approval; } \\
\text { 16.3. Vicarious } \\
\text { consequences } \\
\text { Control group did not } \\
\text { see the video or have } \\
\text { the discussion }\end{array}$ & $\begin{array}{l}\text { No } \\
\text { significant } \\
\text { differences }\end{array}$ \\
\hline $\begin{array}{l}\text { Tani et al. } \\
(2021) . \\
\text { Italy }\end{array}$ & $\begin{array}{l}\text { Tested a gam- } \\
\text { bling interven- } \\
\text { tion for students } \\
\text { through training } \\
\text { teachers about } \\
\text { gambling }\end{array}$ & $\begin{array}{l}393 \text { students } \\
\text { Age: } \\
\text { mean }=\mathrm{nr} ; \\
\text { range }=13-19 \\
\text { Gender: } \\
\text { male }=84 \% ; \\
\text { female }=16 \%\end{array}$ & $\begin{array}{l}\text { Outcome }(s) \text { : } \\
\text { gambling } \\
\text { frequency; gam- } \\
\text { bling problems } \\
\text { Tool }(s) \text { : } \\
\text { SOGS-RA } \\
\text { Follow-up: } \\
7 \text { months } \\
\text { post-intervention }\end{array}$ & $\begin{array}{l}\text { Delivery mode: face- } \\
\text { to face; computer } \\
\text { Provider: teacher } \\
\text { Intensity and dura- } \\
\text { tion: } 16 \text { hours, weeks } \\
\text { ns } \\
\text { BCTs used: } 4.2 \text {. } \\
\text { Information about } \\
\text { antecedents } \\
\text { Control group attend- } \\
\text { ed classes which no } \\
\text { teacher had attended } \\
\text { training }\end{array}$ & $\begin{array}{l}\text { Significant } \\
\text { decrease in } \\
\text { SOGS-RA } \\
\text { scores in } \\
\text { interven- } \\
\text { tion but not } \\
\text { control }\end{array}$ \\
\hline
\end{tabular}


Table 1 (continued)

\begin{tabular}{|c|c|c|c|c|c|}
\hline $\begin{array}{l}\text { Gen- } \\
\text { eral study } \\
\text { information }\end{array}$ & Aims & Participants & Measures & Intervention & Findings \\
\hline $\begin{array}{l}\text { Turner et al. } \\
\text { (2008a, study } \\
\text { 2). Canada }\end{array}$ & $\begin{array}{l}\text { Evaluated a } \\
\text { school-based } \\
\text { educational gam- } \\
\text { bling prevention } \\
\text { curriculum }\end{array}$ & $\begin{array}{l}201 \text { high } \\
\text { school } \\
\text { students } \\
\text { Age: } \\
\text { mean=nr; } \\
\text { range=15-19 } \\
\text { Gender: } \\
\text { male =66; } \\
\text { female }=135\end{array}$ & $\begin{array}{l}\text { Outcome }(s) \text { : } \\
\text { gambling } \\
\text { problems } \\
\text { Tool }(s): \\
\text { SOGS-RA } \\
\text { Time: } 4-5 \text { weeks } \\
\text { post-intervention }\end{array}$ & $\begin{array}{l}\text { Delivery mode: } \\
\text { face-to face; playable } \\
\text { electronic storage } \\
\text { Provider: teacher } \\
\text { Intensity and dura- } \\
\text { tion: } 7 \mathrm{x} \sim 70 \text { min, } \\
\text { once per week } \\
\text { BCTs used: } 2.3 \text {. } \\
\text { Self-monitoring } \\
\text { of behavior; } 4.2 \text {. } \\
\text { Information about } \\
\text { antecedents; } 4.4 \text {. Be- } \\
\text { havioral experiments; } \\
\text { 5.1 Information about } \\
\text { health consequences; } \\
\text { 5.3. Information about } \\
\text { social and environ- } \\
\text { mental consequences; } \\
\text { 5.6. Information } \\
\text { about emotional } \\
\text { consequences } \\
\text { Control group com- } \\
\text { pleted assessments } \\
\text { only }\end{array}$ & $\begin{array}{l}\text { No } \\
\text { significant } \\
\text { differences }\end{array}$ \\
\hline
\end{tabular}


Table 1 (continued)

\begin{tabular}{|c|c|c|c|c|c|}
\hline $\begin{array}{l}\text { Gen- } \\
\text { eral study } \\
\text { information }\end{array}$ & Aims & Participants & Measures & Intervention & Findings \\
\hline $\begin{array}{l}\text { Turner et al. } \\
(2008 b) . \\
\text { Canada }\end{array}$ & $\begin{array}{l}\text { Evaluated a } \\
\text { 1-hour gambling } \\
\text { prevention } \\
\text { program for } \\
\text { students }\end{array}$ & $\begin{array}{l}374 \text { students } \\
\text { in grades 5-12 } \\
\text { Age: } \mathrm{nr} \\
\text { Gender: } \mathrm{nr}\end{array}$ & $\begin{array}{l}\text { Measure- } \\
\text { ments: problem } \\
\text { gambling } \\
\text { Tool(s): } \\
\text { SOGS-RA } \\
\text { Follow-up: } \\
7 \text { weeks } \\
\text { post-intervention }\end{array}$ & $\begin{array}{l}\text { Delivery mode: face- } \\
\text { to face; computer } \\
\text { Provider: researcher } \\
\text { Intensity and dura- } \\
\text { tion: } 1 \times 1 \mathrm{~h} \\
\text { BCTs used: } 1.2 \text {. } \\
\text { Problem solving; } 4.2 \text {. } \\
\text { Information about } \\
\text { antecedents; } 4.4 . \text { Be- } \\
\text { havioral experiments; } \\
\text { 5.6. Information about } \\
\text { emotional conse- } \\
\text { quences; } 16.3 \text {. Vicari- } \\
\text { ous consequences } \\
\text { Control group com- } \\
\text { pleted assessments } \\
\text { only }\end{array}$ & $\begin{array}{l}\text { No } \\
\text { significant } \\
\text { differences }\end{array}$ \\
\hline $\begin{array}{l}\text { Walther et } \\
\text { al. (2013). } \\
\text { Germany }\end{array}$ & $\begin{array}{l}\text { Evaluated the } \\
\text { effects of a } \\
\text { school-based } \\
\text { media education } \\
\text { program }\end{array}$ & $\begin{array}{l}2,109 \text { sixth- } \\
\text { and seventh- } \\
\text { grade students } \\
\text { Age: } \\
\text { mean }=12 \\
\text { Gender: } \\
\text { male }=50.4 \% \text {; } \\
\text { female }=49.6 \%\end{array}$ & $\begin{array}{l}\text { Outcome }(s) \text { : } \\
\text { gambling fre- } \\
\text { quency; lifetime } \\
\text { gambling } \\
\text { Tool }(s) \text { : research- } \\
\text { er-generated } \\
\text { questions } \\
\text { Time: } 7 \text { weeks } \\
\text { post-intervention }\end{array}$ & $\begin{array}{l}\text { Delivery mode: face- } \\
\text { to face } \\
\text { Provider: teacher } \\
\text { Intensity and dura- } \\
\text { tion: } 1 \times 90 \text { min } \\
\text { BCTs used: } 4.2 \text {. } \\
\text { Information about } \\
\text { antecedents; } 4.4 \text {. Be- } \\
\text { havioral experiments; } \\
\text { 5.3. Information about } \\
\text { social and environ- } \\
\text { mental consequences; } \\
\text { 5.6. Information } \\
\text { about emotional } \\
\text { consequences } \\
\text { Control group at- } \\
\text { tended regular classes }\end{array}$ & $\begin{array}{l}\text { Significant } \\
\text { decrease } \\
\text { in current } \\
\text { gambling } \\
\text { in inter- } \\
\text { vention } \\
\text { condition } \\
\text { compared } \\
\text { to control. } \\
\text { No change } \\
\text { in lifetime } \\
\text { gambling }\end{array}$ \\
\hline
\end{tabular}


Table 1 (continued)

\begin{tabular}{|c|c|c|c|c|c|}
\hline $\begin{array}{l}\text { Gen- } \\
\text { eral study } \\
\text { information }\end{array}$ & Aims & Participants & Measures & Intervention & Findings \\
\hline $\begin{array}{l}\text { Williams } \\
\text { et al. } \\
\text { (2010). } \\
\text { Canada }\end{array}$ & $\begin{array}{l}\text { Evaluated a } \\
\text { school-based } \\
\text { gambling pre- } \\
\text { vention program }\end{array}$ & $\begin{array}{l}1,686 \text { high } \\
\text { school } \\
\text { students } \\
\text { Age: } \\
\text { mean }=16 ; \\
\text { range }=14-20 \\
\text { Gender: } \\
\text { male }=53 \%\end{array}$ & $\begin{array}{l}\text { Outcome }(s) \text { : } \\
\text { gambling } \\
\text { frequency; } \\
\text { gambling expen- } \\
\text { diture; gambling } \\
\text { problems } \\
\text { Tool(s): } \\
\text { researcher-gen- } \\
\text { erated questions; } \\
\text { DSM-IV-MR-J } \\
\text { Follow-up: } \\
\text { 3-7 months } \\
\text { post-intervention }\end{array}$ & $\begin{array}{l}\text { Two intervention } \\
\text { conditions: standard } \\
\text { and booster } \\
\text { Delivery mode: face- } \\
\text { to face; computer; } \\
\text { playable electronic } \\
\text { storage } \\
\text { Provider: research } \\
\text { assistants } \\
\text { Intensity and dura- } \\
\text { tion: standard: } 5 \text { x } \\
\sim 100 \text { min, over } 2 \\
\text { weeks; booster: ad- } \\
\text { ditional } 1 \text { session } \\
\text { BCTs used: } 3.1 \text {. Social } \\
\text { support (unspecified); } \\
4.1 . \text { Instruction on } \\
\text { how to perform the } \\
\text { behavior; } 4.2 \text {. Infor- } \\
\text { mation about anteced- } \\
\text { ents; } 5.6 \text {. Information } \\
\text { about emotional } \\
\text { consequences } \\
\text { Control group com- } \\
\text { pleted assessments } \\
\text { only }\end{array}$ & $\begin{array}{l}\text { Gambling } \\
\text { frequency } \\
\text { sig- } \\
\text { nificantly } \\
\text { decreased } \\
\text { in the } \\
\text { standard } \\
\text { and booster } \\
\text { conditions } \\
\text { but not the } \\
\text { control. No } \\
\text { significant } \\
\text { decrease } \\
\text { in problem } \\
\text { gam- } \\
\text { bling or } \\
\text { gambling } \\
\text { expenditure }\end{array}$ \\
\hline
\end{tabular}

Note: Addiction Severity Index-Gambling: ASI-G; Canadian Adolescent Gambling Inventory: CAGI; Cognitive behavioral intervention: CBI; Cognitive behavioral therapy: CBT; DSM-IV-Multiple ResponseJuvenile: DSM-IV-MR-J; Education: EDU; Gambling Activities Questionnaire: GAQ; Gambling Problem Index: GPI; Gambling Quantity and Perceived Norms Scale: GQPN; Gambling Timeline Followback: G-TLFB; Motivational enhancement therapy: MET; not reported: nr; Personalized feedback intervention: PFI; South Oaks Gambling Screen-revised for Adolescents: SOGS-RA

diately post-intervention to 9 months post-intervention. In terms of behavior change, 11/16 $(69 \%)$ interventions demonstrated significant reductions in gambling behavior.

As is shown in Table 2, three studies were classified as low risk of bias, thirteen studies had some concerns of risk, and no studies were considered high risk. Ten concerns related to missing outcomes, six to the randomization process and deviation from intended interventions, five to selection of the reported result, and three to measurement of the outcome. The results should therefore be interpreted with caution.

\section{BCTs and MOD}

A total of 18 different BCTs were adopted in the intervention or control conditions across all studies (see supplementary material 1 for the BCTs included in each study). The average number of BCTs per study was 4 , with a range of 1 to 10 . All 18 BCTs were present in the intervention condition and two techniques were identified in the control conditions. With regards to the latter, a single technique was adopted in two controls ('2.2. Feedback on behavior' and '4.2. Information about antecedents'). Note that the study including '2.2. 
Table 2 Quality assessment

\begin{tabular}{|c|c|c|c|c|c|c|}
\hline Paper & $\begin{array}{l}\text { Random- } \\
\text { ization } \\
\text { process }\end{array}$ & $\begin{array}{l}\text { Deviation } \\
\text { from intended } \\
\text { interventions }\end{array}$ & $\begin{array}{l}\text { Missing } \\
\text { outcome } \\
\text { data }\end{array}$ & $\begin{array}{l}\text { Measure- } \\
\text { ment } \\
\text { of the } \\
\text { outcome }\end{array}$ & $\begin{array}{l}\text { Selection } \\
\text { of the } \\
\text { reported } \\
\text { result }\end{array}$ & $\begin{array}{l}\text { Over- } \\
\text { all }\end{array}$ \\
\hline Broussard \& Wulfert (2017) & + & + & + & + & + & + \\
\hline Calado et al. (2020) & + & $?$ & $?$ & $?$ & $?$ & $?$ \\
\hline Canale et al. (2016) & + & + & $?$ & + & + & $?$ \\
\hline Donati et al. (2018, study 2) & + & $?$ & + & + & $?$ & $?$ \\
\hline Donati et al. (2014) & + & + & $?$ & + & $?$ & $?$ \\
\hline Gaboury \& Ladouceur (1993) & $?$ & + & $?$ & $?$ & $?$ & $?$ \\
\hline Huic et al. (2017) & + & $?$ & $?$ & $?$ & + & $?$ \\
\hline Larimer et al. (2012) & $?$ & + & + & + & + & $?$ \\
\hline Martens et al. (2015) & + & + & + & + & + & + \\
\hline Petry et al. (2009) & + & + & + & + & + & + \\
\hline St-Pierre et al. (2017) & $?$ & + & $?$ & + & + & $?$ \\
\hline Tani et al. (2021) & + & $?$ & $?$ & + & + & $?$ \\
\hline Turner et al. (2008a, study 2) & $?$ & + & + & + & + & $?$ \\
\hline Turner et al. (2008b) & $?$ & $?$ & $?$ & + & + & $?$ \\
\hline Walther et al. (2013) & + & + & $?$ & + & + & $?$ \\
\hline Williams et al. (2010) & $?$ & $?$ & $?$ & + & $?$ & $?$ \\
\hline
\end{tabular}

Note $+=$ low risk of bias; $?=$ some concerns

Table 3 Frequency of BCTs in intervention conditions

\begin{tabular}{|c|c|c|c|c|}
\hline \multirow[b]{2}{*}{ BCT code \& label } & \multicolumn{2}{|c|}{$\begin{array}{l}\text { BCT in all } \\
\text { interventions } \\
(\mathrm{Max}=16)\end{array}$} & \multicolumn{2}{|c|}{$\begin{array}{l}\text { BCT in effective } \\
\text { interventions } \\
(\mathrm{Max}=11)\end{array}$} \\
\hline & $n$ & $\%$ & $n$ & $\%$ \\
\hline 1.2. Problem solving & 4 & 25 & 0 & 0 \\
\hline 2.2. Feedback on behavior & 3 & 19 & 3 & 27 \\
\hline 2.3. Self-monitoring of behavior & 2 & 13 & 0 & 0 \\
\hline 3.1. Social support (unspecified) & 3 & 19 & 3 & 27 \\
\hline 4.1. Instruction on how to perform the behavior & 4 & 25 & 1 & 9 \\
\hline 4.2. Information about antecedents & 11 & 69 & $7 *$ & 64 \\
\hline 4.4. Behavioral experiments & 7 & 44 & $5 *$ & 45 \\
\hline 5.1. Information about health consequences & 4 & 25 & 0 & 0 \\
\hline 5.3. Information about social and environmental consequences & 9 & 56 & $5 *$ & 45 \\
\hline 5.6. Information about emotional consequences & 9 & 56 & $5 *$ & 45 \\
\hline 6.1. Demonstration of the behavior & 1 & 6 & 0 & 0 \\
\hline 6.2. Social comparison & 3 & 19 & 1 & 9 \\
\hline 6.3. Information about others' approval & 1 & 6 & 0 & 0 \\
\hline 8.1. Behavioral practice/rehearsal & 2 & 13 & 0 & 0 \\
\hline 9.1. Credible source & 1 & 6 & 0 & 0 \\
\hline 9.2. Pros and cons & 1 & 6 & 1 & 9 \\
\hline 14.2. Punishment & 1 & 6 & 1 & 9 \\
\hline 16.3. Vicarious experiences & 3 & 19 & 0 & 0 \\
\hline
\end{tabular}

Note: *Promising BCTs (identified as being present in $\geq 25 \%$ of all interventions and in two effective interventions) 
Feedback on behavior' in the control also included the technique in the intervention condition. The use of the technique was therefore excluded for that study. In relation to the intervention conditions, the most frequently used BCT was '4.2. Information about antecedents', which was present in 11/16 (69\%) of interventions. Other commonly adopted BCTs were '5.3. Information about social and environmental consequences' $(9 / 16,56 \%)$, '5.6. Information about emotional consequences' $(9 / 16,56 \%)$, and '4.4. Behavioral experiments' (7/16, $44 \%)$.

In terms of effectiveness, Table 3 shows four BCTs were labeled 'promising'. That is, those $\mathrm{BCT}$ s present in at least $25 \%$ of all interventions, in at least two effective interventions,

Table 4 Definitions of identified BCTs

BCT code \& label Definition

1.2. Problem solving

Analyse, or prompt the person to analyse, factors influencing the behavior and generate or select strategies that include overcoming barriers and/or increasing facilitators

\subsection{Feedback on behavior}

2.3. Self-monitoring of behavior

3.1. Social support (unspecified)

4.1. Instruction on how to perform the behavior

4.2. Information about antecedents

\subsection{Behavioral experiments}

5.1. Information about health consequences

5.3. Information about social and environmental consequences

5.6. Information about emotional consequences

6.1. Demonstration of the behavior

6.2. Social comparison

6.3. Information about others' approval

8.1. Behavioral practice/rehearsal

9.1. Credible source

9.2. Pros and cons

14.2. Punishment

16.3. Vicarious consequences
Monitor and provide informative or evaluative feedback on performance of the behavior

Establish a method for the person to monitor and record their behavior(s) as part of a behavior change strategy

Advise on, arrange or provide social support or non-contingent praise or reward for performance of the behavior

Advise or agree on how to perform the behavior

Provide information about antecedents that reliably predict performance of the behavior

Advise on how to identify and test hypotheses about the behavior, its causes and consequences, by collecting and interpreting data

Provide information about health consequences of performing the behavior

Provide information about social and environmental consequences of performing the behavior

Provide information about emotional consequences of performing the behavior

Provide an observable sample of the performance of the behavior, directly in person or indirectly

Draw attention to others' performance to allow comparison with the person's own performance

Provide information about what other people think about the behavior. The information clarifies whether others will like, approve or disapprove of what the person is doing or will do

Prompt practice or rehearsal of the performance of the behavior one or more times in a context or at a time when the performance may not be necessary, in order to increase habit and skill

Present verbal or visual communication from a credible source in favor of or against the behavior

Advise the person to identify and compare reasons for wanting (pros) and not wanting to (cons) change the behavior

Arrange for aversive consequence contingent on the performance of the unwanted behavior

Prompt observation of the consequences for others when they perform the behavior 
and not included in both intervention and control conditions. These were '4.2. Information about antecedents' (7/11, 64\%), ‘4.4. Behavioral experiments' (5/11, 45\%), '5.3. Information about social and environmental consequences' $(9 / 11,45 \%)$, and '5.6. Information about emotional consequences' (9/11, 45\%). Definitions of BCTs can be seen in Table 4.

The reviewed studies contained a total of six MODs: face-to-face; website; computer; playable electronic storage (i.e., video tapes, DVDs); printed publication; and video game (see Table 5). Most interventions were delivered using two MODs $(n=8)$, whereas six interventions used a single MOD and two interventions used three MODs. The delivery mode used most frequently was face-to-face $(14 / 16,88 \%)$, followed by playable electronic storage $(5 / 16,31 \%)$, and computer $(4 / 16,25 \%)$. The MODs labeled as promising were faceto-face $(9 / 11,82 \%)$, computer $(3 / 11,27 \%)$, and playable electronic storage $(2 / 11,18 \%)$. Definitions of MODs can be seen in Table 6.

\section{Discussion}

The systematic review identified the BCTs and MODs adopted in interventions targeting adolescent gambling behavior. Sixteen studies met the inclusion criteria, eleven of which successfully changed gambling behavior.

Table 5 Frequency of MODs used in intervention conditions

\begin{tabular}{lllll}
\hline & $\begin{array}{l}\text { MOD in all interventions } \\
(\text { Max =16) }\end{array}$ & $\begin{array}{l}\text { MOD in effective } \\
\text { interventions } \\
\text { (Max=11) }\end{array}$ & $\%$ \\
\hline MOD & $n$ & $\%$ & $9 *$ & 82 \\
Face-to face & 14 & 88 & 1 & 9 \\
Website & 1 & 6 & $3 *$ & 27 \\
Computer & 4 & 25 & $2 *$ & 18 \\
Playable electronic storage & 5 & 31 & 3 & 27 \\
Printed publication & 3 & 6 & 1 & 9 \\
Video game & 1 & 6 & 9
\end{tabular}

Note: *Promising MODs (identified as being present in $\geq 25 \%$ of all interventions and in two effective interventions)

Table 6 Definitions of identified MODs

\begin{tabular}{ll}
\hline Mode of Delivery & Definition \\
\hline Computer & $\begin{array}{l}\text { Electronic mode of delivery that involves presentation of information by a } \\
\text { desktop or laptop computer } \\
\text { Fuce-to face }\end{array}$ \\
$\begin{array}{l}\text { Human interactional mode of delivery that involves an intervention source } \\
\text { and recipient being together in the same location and communicating directly }\end{array}$ \\
Playable electronic storage & $\begin{array}{l}\text { Electronic mode of delivery that involves presentation of information stored } \\
\text { on an object that is inserted into a playing device }\end{array}$ \\
Printed publication & $\begin{array}{l}\text { Printed material mode of delivery that involves use of a printed publication } \\
\text { Electronic mode of delivery that involves the intervention recipient playing } \\
\text { a computer game }\end{array}$ \\
Website & $\begin{array}{l}\text { Electronic mode of delivery that involves the intervention recipient interact- } \\
\text { ing with a website }\end{array}$ \\
\hline
\end{tabular}


The review found a range of BCTs have been included in adolescent gambling interventions. The BCTs most frequently adopted were educational with strategies attempting to inform adolescents about the antecedents and consequences (emotional, social, and environmental) of problem gambling. For example, Tani et al. (2021) gave information on various problem gambling risk factors and Donati et al. (2014) presented participants with the economic disadvantages associated with gambling. The adoption of such BCTs is likely due to the knowledge within the target population and the purpose of the interventions. Specifically, knowledge of gambling, its potential consequences and other related cognitions may be lacking or erroneous in adolescents (St-Pierre et al., 2015). Such interventions therefore use these BCTs to correct beliefs or introduce new information, in the hope that cognition change influences gambling participation (see Keen et al., 2019). In terms of effectiveness, these BCTs were also three of the four labeled promising. Current findings are consistent with studies demonstrating preliminary effectiveness of educational interventions in reducing gambling behavior (Forsström et al., 2021). Thus, future interventions designed to modify adolescent gambling behavior should seek to include the BCTs '4.2. Information about antecedents', '5.3. Information about social and environmental consequences', and '5.6. Information about emotional consequences'. The final promising technique involved behavioral experiments, wherein participants simulate gambling and experience the immediate consequences in a controlled environment. For example, Calado et al. (2020) demonstrated randomness by having students play and bet on a roulette. Similarly, Broussard and Wulfert (2017) had participants play a slot machine programmed to demonstrate monetary losses over time. Through participation in gambling and experiencing negative consequences, such interventions attempt to dissuade future participation in the behavior. The review therefore suggests that, in addition to the aforementioned three BCTs, interventions should, at a minimum, consider adopting the technique '4.4. Behavioral experiments'. Interventions including these four BCTs could successfully demonstrate a reduction in adolescent gambling behavior.

In relation to the MODs, a range of delivery modes were adopted within the interventions. Most interventions included the face-to-face modality. The use of this delivery mode is again perhaps due to the target population and the potential reach of the setting. Indeed, educational settings where adolescents regularly attend, such as school, college, and university, provide an ideal opportunity for face-to-face intervention delivery. For example, Walther et al. (2013) trained teachers to deliver the intervention face-to-face to participants during class time. Other frequent MODs included technologies such as computers and playable electronic storage; however, these were always combined with a face-to-face component. For example, Williams et al.'s (2010) intervention was delivered mostly in person but utilized a computer to deliver PowerPoint slides. In terms of effectiveness, successful interventions were delivered using these three most frequently adopted MODs. Intervention developers should therefore look to computer, playable electronic storage, and/or faceto-face methods for content delivery, which mirror the playing platforms that adolescents gamble on.

The review identifies the BCTs and MODs most likely to reduce adolescent gambling behavior. However, there may be additional opportunities for intervention developers. Some of the effective BCTs identified by Humphreys et al. (2021) were not used often in our review. For example, '2.3. Self-monitoring of behavior' was only included in two interventions. This technique has also shown effectiveness in changing other health-related behav- 
iors such as sedentariness (Compernolle et al., 2019) and alcohol consumption (Crane et al., 2018). Additionally, from the 93 BCTs included in the BCTTv1 taxonomy, we found $75(81 \%)$ were not included in any intervention. This suggests that gambling interventions for adolescents have adopted minimal techniques and designers have at their disposal many other strategies that could be useful. Of course, not all untapped BCTs will be effective and it is up to researchers to establish effectiveness. We recommend new programs adopt the promising BCTs identified here whilst exploratory and experimental work establishes how effective the additional techniques are in modifying adolescent gambling behavior.

In terms of the MODs, other modes exist for intervention delivery. This could be especially useful given the challenges of face-to-face delivery during the outbreak of the COVID-19 pandemic (Quail et al., 2021). Mobile phones have shown to be effective in promoting other health behaviors (Yang \& Van Stee, 2019). This MOD may be particularly appealing given the relative cheapness and significant reach of mobile interventions, and the high usage of mobile phones amongst adolescents (Lopez-Fernandez et al., 2014). Additionally, some of the delivery modes that were effective in interventions but not frequently used could prove fruitful. For example, the use of a website, which was only adopted by Canale et al. (2016), showed significant intervention effects in reducing gambling behavior. Similarly, printed publications were not adopted frequently $(n=3)$, yet all interventions including the delivery mode were effective.

It is interesting to note the frequency of BCTs included in interventions. Research synthesizing BCT frequency has found increased effectiveness when interventions include a greater number of BCTs (Webb et al., 2010). However, adopting multiple BCTs does not always lead to effective interventions (Bohlen et al., 2020). In the present study, two interventions were effective whilst using a single BCT whereas successful change was also seen in an intervention using 10 BCTs (albeit the latter may find difficulty in identifying the main change agent). Moreover, some interventions adopting the same number of BCTs showed different effects. Instead of focusing on BCT frequency, it is more important to consider how techniques combine or interact. Techniques may have a synergistic or additive effect, or they may nullify the effects of others (Dusseldorp et al., 2014). Therefore, although we identify the BCTs apparent in interventions, that is not to say each technique contributed equally to effectiveness. However, using the promising BCTs could be a useful starting point and future research should establish the optimal frequency and combinations of techniques. Another consideration is isolating the effective BCTs when they are delivered in combination with ineffective ones. A single component intervention with equal effectiveness as a multi-component intervention is more desirable from both an individual experiential perspective and an economic one.

We also note that, consistent with Keen et al. (2017), only a small number of studies reported measures of behavioral outcomes and instead focused on cognitions. As such, some studies targeting and measuring gambling cognitions only were excluded, some of which were effective in changing such cognitions. For example, Zhou et al. (2019) found that a GameSense prevention program positively manipulated knowledge about gambling and intentions towards gambling in the future. The primary focus on cognitions could be due to difficulty in obtaining behavioral measures (Braverman et al., 2014) or because researchers assume successful change will lead to behavior change. However, although interventions may change cognitions, they have not always managed to change actual gambling behavior (Williams et al., 2012b). Thus, assessing cognition change is no proxy for behavior change, 
despite being a necessary first step. Another reason could be due to the sample studied; adolescents and young adults are not legally allowed to gamble. However, given gambling rates in this group (Calado et al., 2017; Emond et al., 2020), interventions should not only measure and modify gambling beliefs, but attention should also be given to actual gambling behavior.

\section{Limitations}

There are some limitations to note. First, intervention success depends on other factors aside from the content and delivery mode. For example, the fidelity of delivery can determine whether an intervention is effective (Bellg et al., 2004) and multiple factors can influence intervention uptake (Milat et al., 2013). Second, the approach to identifying 'promising' BCTs may have some attached limitations. For example, the usefulness of a technique used often but showing success on only two occasions could be questioned. However, there is no agreed method for identifying effective BCTs and each approach used to date has limitations (Michie et al., 2018). Following previous work (e.g., Ahmed et al., 2021; Brown et al., 2019, 2020; Lorencatto et al., 2012), the approach used has the potential to identify BCTs that could be effective. Third, some studies only had short-term follow-up meaning it is unclear whether initial behavior change was sustained over time. Interventions should therefore assess intervention effects over a longer period. Fourth, BCT identification relies heavily on accurate reporting in studies. Techniques would be missed in the extraction process if, for example, they were either reported incorrectly, reported vaguely, or not reported at all. As has been noted in other work (e.g., Glasziou et al., 2008; Scott et al., 2020), reporting of intervention content was lacking in some studies. If word counts prevent detailed reports of materials, supplementary files should be used to make intervention content explicit. Finally, the identified studies were limited by the databases used and the inclusion of studies in English language only.

\section{Conclusions}

The study reviewed the content of interventions implemented to reduce adolescent gambling behavior. The review findings highlight four specific BCTs that were more effective than others at reducing the behavior. Additionally, the review found that three delivery modes were apparent in successful interventions. Given these findings, we recommend developers strongly consider incorporating these when designing new interventions for this population. The range of BCTs and MODs used across studies was also relatively narrow compared to other areas of behavior change. Future experimentation with BCTs and MODs not represented in the current review is needed, to enhance the efficacy of adolescent harm prevention programs more broadly.

Supplementary Information The online version contains supplementary material available at https://doi. org/10.1007/s10899-022-10108-8.

Funding No funding was received for conducting this study.

Declarations The authors have no relevant financial or non-financial interests to disclose. 


\section{References}

Ahmed, S., Heaven, A., Lawton, R., Rawlings, G., Sloan, C., \& Clegg, A. (2021). Behaviour change techniques in personalised care planning for older people: a systematic review. British Journal of General Practice, 71(703), e121-e127. https://doi.org/10.3399/bjgp20X714017

Armitage, R. (2021). Gambling among adolescents: An emerging public health problem. The Lancet. Public Health, 6(3), e143. https://doi.org/10.1016/S2468-2667(21)00026-8

Bellg, A. J., Borrelli, B., Resnick, B., Hecht, J., Minicucci, D. S., Ory, M. .. Czajkowski, S. (2004). Enhancing treatment fidelity in health behavior change studies: Best practices and recommendations from the NIH Behavior Change Consortium. Health Psychology, 23(5), 443-451. https://doi. org/10.1037/0278-6133.23.5.443

Bohlen, L. C., Michie, S., de Bruin, M., Rothman, A. J., Kelly, M. P., Groarke, H. ... Johnston, M. (2020). Do combinations of behavior change techniques that occur frequently in interventions reflect underlying theory? Annals of Behavioral Medicine, 54(11), 827-842. https://doi.org/10.1093/abm/kaaa078

Braverman, J., Tom, M. A., \& Shaffer, H. J. (2014). Accuracy of self-reported versus actual online-gambling wins and losses. Psychological Assessment, 26, 865-877

Broussard, J., \& Wulfert, E. (2017). Can an accelerated gambling simulation reduce persistence on a gambling task? International Journal of Mental Health and Addiction, 15(1), 143-153. https://doi.org/10.1007/ s11469-015-9620-8

Brown, T., Hardeman, J., Bauld, W., Holland, L., Maskrey, R., Naughton, V. ... Notley, C. (2019). A systematic review of behaviour change techniques within interventions to prevent return to smoking postpartum. Addictive Behaviors, 92, 236-243. https://doi.org/10.1016/j.addbeh.2018.12.031

Brown, T. J., Gentry, S., Bauld, L., Boyle, E. M., Clarke, P., Hardeman, W. ... Notley, C. (2020). Systematic review of behaviour change techniques within interventions to reduce environmental tobacco smoke exposure for children. International Journal of Environmental Research and Public Health, 17(21), 7731. https://doi.org/10.3390/ijerph17217731

Burge, A. N., Pietrzak, R. H., Molina, C. A., \& Petry, N., M (2004). Age of gambling initiation and severity of gambling and health problems among older adult problem gamblers. Psychiatric Services, 55, 1437-1439

Caillon, J., Grall-Bronnec, M., Perrot, B., Leboucher, J., Donnio, Y., Romo, L., \& Challet-Bouju, G. (2019). Effectiveness of at-risk gamblers' temporary self-exclusion from internet gambling sites. Journal of Gambling Studies, 35(2), 601-615. https://doi.org/10.1007/s10899-018-9782-y

Calado, F., Alexandre, J., \& Griffiths, M. D. (2017). Prevalence of adolescent problem gambling: a systematic review of recent research. Journal of Gambling Studies, 33, 397-424

Calado, F., Alexandre, J., Rosenfeld, L., Pereira, R., \& Griffiths, M. D. (2020). The efficacy of a gambling prevention program among high-school students. Journal of Gambling Studies, 36(2), 573-595. https:// doi.org/10.1007/s10899-019-09908-2

Canale, N., Vieno, A., Griffiths, M. D., Marino, C., Chieco, F., Disperati, F. ... Santinello, M. (2016). The efficacy of a web-based gambling intervention program for high school students: A preliminary randomized study. Computers in Human Behavior, 55, 946-954. https://doi.org/10.1016/j.chb.2015.10.012

Compernolle, S., DeSmet, A., Poppe, L., Crombez, G., De Bourdeaudhuij, I., Cardon, G. ... Van Dyck, D. (2019). Effectiveness of interventions using self-monitoring to reduce sedentary behavior in adults: A systematic review and meta-analysis. International Journal of Behavioral Nutrition and Physical Activity, 16(1), 63. https://doi.org/10.1186/s12966-019-0824-3

Cook, S., Turner, N. E., Ballon, B., Paglia-Boak, A., Murray, R., Adlaf, E. M. .. Mann, R. E. (2015). Problem gambling among Ontario students: Associations with substance abuse, mental health problems, suicide attempts, and delinquent behaviours. Journal of Gambling Studies, 31(4), 1121-1134. https:// doi.org/10.1007/s10899-014-9483-0

Crane, D., Garnett, C., Michie, S., West, R., \& Brown, J. (2018). A smartphone app to reduce excessive alcohol consumption: Identifying the effectiveness of intervention components in a factorial randomised control trial. Scientific Reports, 8(1), 4384

Delfabbro, P. H., Winefield, A. H., \& Anderson, S. (2009). Once a gambler-Always a gambler? A longitudinal analysis of gambling patterns in young people making the transition from adolescence to adulthood. International Gambling Studies, 9(2), 151-163. https://doi.org/10.1080/14459790902755001

Delfabbro, P., \& King, D. L. (2020). Gaming-gambling convergence: Evaluating evidence for the 'gateway' hypothesis. International Gambling Studies, 20(3), 380-392. https://doi.org/10.1080/14459795.2020. 1768430

Derevensky, J., \& Gilbeau, L. (2015). Adolescent gambling: Twenty-five years of research. Canadian Journal of Addiction/Le Journal Canadien d'Addiction, 6, 4-12 
Dombrowski, S. U., O’Carroll, R. E., \& Williams, B. (2016). Form of delivery as a key 'active ingredient' in behaviour change interventions. British Journal of Health Psychology, 21(4), 733-740

Donati, M. A., Primi, C., \& Chiesi, F. (2014). Prevention of problematic gambling behavior among adolescents: Testing the efficacy of an integrative intervention. Journal of Gambling Studies, 30(4), 803-818. https://doi.org/10.1007/s10899-013-9398-1

Donati, M. A., Chiesi, F., Iozzi, A., Manfredi, A., Fagni, F., \& Primi, C. (2018). Gambling-related distortions and problem gambling in adolescents: A model to explain mechanisms and develop interventions. Frontiers in Psychology, 8, 2243. https://doi.org/10.3389/fpsyg.2017.02243

Dowling, N. A., Merkouris, S. S., Greenwood, C. J., Oldenhof, E., Toumbourou, J. W., \& Youssef, G. J. (2017). Early risk and protective factors for problem gambling: A systematic review and meta-analysis of longitudinal studies. Clinical Psychology Review, 51, 109-124. https://doi.org/10.1016/j.cpr.2016.10.008

Dusseldorp, E., van Genugten, L., van Buuren, S., Verheijden, M. W., \& van Empelen, P. (2014). Combinations of techniques that effectively change health behavior: Evidence from Meta-CART analysis. Health Psychology, 33(12), 1530-1540

Emond, A., Griffiths, M. D., \& Hollén, L. (2020). Problem gambling in early adulthood: A population-based study. International Journal of Mental Health and Addiction. https://oi.org/10.1007/ s11469-020-00401-1

Forsström, D., Spångberg, J., Petterson, A., Brolund, A., \& Odeberg, J. (2021). A systematic review of educational programs and consumer protection measures for gambling: An extension of previous reviews. Addiction Research \& Theory, 29(5), 398-412. https://doi.org/10.1080/16066359.2020.1729753

Gaboury, A., \& Ladouceur, R. (1993). Evaluation of a prevention program for pathological gambling among adolescents. Journal of Primary Prevention, 14(1), 21-28. https://doi.org/10.1007/BF01324653

Glasziou, P., Meats, E., Heneghan, C., \& Shepperd, S. (2008). What is missing from descriptions of treatment in trials and reviews? BMJ, 336, 1472

Griffiths, M. D., \& Parke, J. (2010). Adolescent gambling on the Internet: A review. International Journal of Adolescent Medicine and Health, 22, 59-75

Hardoon, K. K., Gupta, R., \& Derevensky, J. L. (2004). Psychosocial variables associated with adolescent gambling. Psychology of Addictive Behaviors, 18(2), 170-179. https://doi.org/10.1037/0893-164X.18.2.170

Hollén, L., Dörner, R., Griffiths, M. D., et al. (2020). Gambling in young adults aged 17-24 years: A populationbased study. Journal of Gambling Studies, 36, 747-766. https://doi.org/10.1007/s10899-020-09948-z

Huic, A., Kranzelic, V., Hundric, D., D., \& Ricijas, N. (2017). Who really wins? Efficacy of a Croatian youth gambling prevention program. Journal of Gambling Studies, 33(3), 1011-1033. https://doi.org/10.1007/ s10899-017-9668-4

Humphreys, G., Evans, R., Makin, H., Cooke, R., \& Jones, A. (2021). Identification of behavior change techniques from successful web-based interventions targeting alcohol consumption, binge eating, and gambling: Systematic review. Journal of Medical Internet Research, 23(2), e22694. https://oi. org/10.2196/22694

Keen, B., Blaszczynski, A., \& Anjoul, F. (2017). Systematic review of empirically evaluated school-based gambling education programs. Journal of Gambling Studies, 33(1), 301-325

Keen, B., Anjoul, F., \& Blaszczynski, A. (2019). How learning misconceptions can improve outcomes and youth engagement with gambling education programs. Journal of Behavioral Addictions, 8(3), 372383. https://doi.org/10.1556/2006.8.2019.56

Kryszajtys, D. T., Hahmann, T. E., Schuler, A., et al. (2018). Problem gambling and delinquent behaviours among adolescents: A scoping review. Journal of Gambling Studies, 34, 893-914. https://doi. org/10.1007/s10899-018-9754-2

Larimer, M. E., Neighbors, C., Lostutter, T. W., Whiteside, U., Cronce, J. M., Kaysen, D., \& Walker, D. D. (2012). Brief motivational feedback and cognitive behavioral interventions for prevention of disordered gambling: A randomized clinical trial. Addiction, 107, 1148-1158. https://doi. org/10.1111/j.1360-0443.2011.03776.x

Livazović, G., \& Bojčić, K. (2019). Problem gambling in adolescents: What are the psychological, social and financial consequences? BMC Psychiatry, 19, 308

Lopez-Fernandez, O., Honrubia-Serrano, L., Freixa-Blanxart., M., \& Gibson, W. (2014). Prevalence of problematic mobile phone use in British adolescents. Cyberpsychology, Behavior, and Social Networking, 17(2), 91-98. https://doi.org/10.1089/cyber.2012.0260

Lorencatto, F., West, R., \& Michie, S. (2012). Specifying evidence-based behavior change techniques to aid smoking cessation in pregnancy. Nicotine \& Tobacco Research, 14(9), 1019-1026

Marques, M. M., Carey, R. N., Norris, E., Evans, F., Finnerty, A. N., Hastings, J. ... Michie, S. (2021). Delivering behaviour change interventions: Development of a mode of delivery ontology. Wellcome Open Research, 5, 125. https://doi.org/10.12688/wellcomeopenres.15906.2 
Martens, M. P., Arterberry, B. J., Takamatsu, S. K., Masters, J., \& Dude, K. (2015). The efficacy of a personalized feedback-only intervention for at-risk college gamblers. Journal of Consulting and Clinical Psychology, 83(3), 494-499. https://doi.org/10.1037/a0038843

Messerlian, C., Byrne, A. M., \& Derevensky, J. L. (2004). Gambling, youth and the internet: Should we be concerned? The Canadian Child and Adolescent Psychiatry Review, 13(1), 3-6

Michie, S., Abraham, C., Whittington, C., McAteer, J., \& Gupta, S. (2009). Effective techniques in healthy eating and physical activity interventions: A meta-regression. Health Psychology, 28(6), 690-701. https://doi.org/10.1037/a0016136

Michie, S., Richardson, M., Johnston, M., Abraham, C., Francis, J., Hardeman, W. ... Wood, C. E. (2013). The behavior change technique taxonomy (v1) of 93 hierarchically clustered techniques: Building an international consensus for the reporting of behavior change interventions. Annals of Behavioral Medicine, 46(1), 81-95. https://doi.org/10.1007/s12160-013-9486-6

Michie, S., West, R., Sheals, K., \& Godinho, C. A. (2018). Evaluating the effectiveness of behavior change techniques in health-related behavior: A scoping review of methods used. Translational Behavioral Medicine, 8(2), 212-224. https://doi.org/10.1093/tbm/ibx019

Milat, A. J., King, L., Bauman, A. E., \& Redman, S. (2013). The concept of scalability: Increasing the scale and potential adoption of health promotion interventions into policy and practice. Health Promotion International, 28(3), 285-298. https://doi.org/10.1093/heapro/dar097

Nowak, D. E., \& Aloe, A. M. (2014). The prevalence of pathological gambling among college students: A meta-analytic synthesis, 2005-2013. Journal of Gambling Studies, 30(4), 819-843. https://doi. org/10.1007/s10899-013-9399-0

Oh, B. C., Ong, Y. J., \& Loo, J. M. Y. (2017). A review of educational-based gambling prevention programs for adolescents. Asian Journal of Gambling Issues and Public Health, 7, 4. https://doi.org/10.1186/ s40405-017-0024-5

Petry, N. M., Weinstock, J., Morasco, B. J., \& Ledgerwood, D. M. (2009). Brief motivational interventions for college student problem gamblers. Addiction, 104(9), 1569-1578. https://oi. org/10.1111/j.1360-0443.2009.02652.x

Proimos, J., DuRant, R., Pierce, H., J. D., \& Goodman, E. (1998). Gambling and other risk behaviors among 8th- to 12th-grade students. Pediatrics, 102(2), 1-6

Quail, Z., Bolton, L., \& Massey, K. (2021). Digital delivery of non-pharmacological intervention programmes for people living with dementia during the COVID-19 pandemic. BMJ Case Reports, 14(6), e242550. https://doi.org/10.1136/bcr-2021-242550

Rodda, S., Merkouris, S. S., Abraham, C., Hodgins, D. C., Cowlishaw, S., \& Dowling, N. A. (2018). Therapist-delivered and self-help interventions for gambling problems: A review of contents. Journal of Behavioral Addictions, 7(2), 211-226. https://doi.org/10.1556/2006.7.2018.44

Scott, C., de Barra, M., Johnston, M., de Bruin, M., Scott, N., Matheson, C. ... Watson, M. C. (2020). Using the behaviour change technique taxonomy v1 (BCTTv1) to identify the active ingredients of pharmacist interventions to improve non-hospitalised patient health outcomes. BMJ Open, 10(9), e036500. https:// doi.org/10.1136/bmjopen-2019-036500

Shaffer, H.J., \& Hall, M.N.(2002). The natural history of gambling and drinking problems among casino employees. The Journal of Social Psychology, 142(4), 405-424. https://doi.org/10.1080/00224540209603909

Shaffer, H. J., \& Korn, D. A. (2002). Gambling and related mental disorders: A public health analysis. Annual Review of Public Health, 23, 171-212

St-Pierre, R. A., Derevensky, J. L., Temcheff, C. E., \& Gupta, R. (2015). Adolescent gambling and problem gambling: Examination of an extended Theory of Planned Behaviour. International Gambling Studies, 15(3), 506-525. https://doi.org/10.1080/14459795.2015.1079640

St-Pierre, R. A., Derevensky, J. L., Temcheff, C. E., Gupta, R., \& Martin-Story, A. (2017). Evaluation of a school-based gambling prevention program for adolescents: Efficacy of using the Theory of Planned Behaviour. Journal of Gambling Issues, 36, 113-137

Sterne, J. A., Savović, J., Page, M. J., Elbers, R. G., Blencowe, N. S., Boutron, I. ... Higgins, J. P. (2019). RoB 2: A revised tool for assessing risk of bias in randomised trials. $B M J, 366,14898$. https://doi. org/10.1136/bmj.14898

Svensson, J., \& Sundqvist, K. (2019). Gambling among Swedish youth: Predictors and prevalence among 15- and 17-year-old students. Nordic Studies on Alcohol and Drugs, 36(2), 177-189. https://doi. org/10.1177/1455072518807788

Tani, F., Ponti, L., Ghinassi, S., \& Smorti, M. (2021). A gambling primary prevention program for students through teacher training: an evidence-based study. International Gambling Studies, 21, 272-294. https://doi.org/10.1080/14459795.2020.1861056

Turner, N. E., Macdonald, J., \& Somerset, M. (2008a). Life skills, mathematical reasoning and critical thinking: A curriculum for the prevention of problem gambling. Journal of Gambling Studies, 24(3), 367380. https://doi.org/10.1007/s10899-007-9085-1 
Turner, N., Macdonald, J., Bartoshuk, M., \& Zangeneh, M. (2008b). The evaluation of a 1-h prevention program for problem gambling. International Journal of Mental Health and Addiction, 6(2), 238-243. https://doi.org/10.1007/s11469-007-9121-5

Volberg, R. A., Gupta, R., Griffiths, M. D., Olason, D. T., \& Delfabbro, P. (2010). An international perspective on youth gambling prevalence studies. International Journal of Adolescent Medicine and Health, 22(1), 3-38

Walther, B., Hanewinkel, R., \& Morgenstern, M. (2013). Short-term effects of a school-based program on gambling prevention in adolescents. Journal of Adolescent Health, 52(5), 599-605. https://doi. org/10.1016/j.jadohealth.2012.11.009

Webb, T. L., Joseph, J., Yardley, L., \& Michie, S. (2010). Using the internet to promote health behavior change: A systematic review and meta-analysis of the impact of theoretical basis, use of behavior change techniques, and mode of delivery on efficacy. Journal of Medical Internet Research, 12(1), e4. https://doi.org/10.2196/jmir.1376

Williams, R. J., Wood, R. T., \& Currie, S. R. (2010). Stacked Deck: An effective, school-based program for the prevention of problem gambling. Journal of Primary Prevention, 31, 109-125. https://doi. org/10.1007/s10935-010-0212-x

Williams, R. J., Volberg, R. A., \& Stevens, R. M. G. (2012a). The population prevalence of problem gambling: Methodological influences, standardized rates, jurisdictional differences, and worldwide trends. Report prepared for the Ontario Problem Gambling Research Centre and the Ontario Ministry of Health and Long Term Care

Williams, R. J., West, B. L., \& Simpson, R. I. (2012b). Prevention of problem gambling: A comprehensive review of the evidence, and identified best practices. [Internet]. Available from: http://hdl.handle. net/10133/3121

Yang, Q., \& Van Stee, S. K. (2019). The comparative effectiveness of mobile phone interventions in improving health outcomes: Meta-analytic review. JMIR Mhealth Uhealth, 7(4), e11244. https://doi. org/10.2196/11244

Zhou, X. L., Goernert, P. N., \& Corenblum, B. (2019). Examining the efficacy of the GameSense gambling prevention programme among university undergraduate students. International Gambling Studies, 19(2), 282-295. https://doi.org/10.1080/14459795.2018.1554083

Publisher's Note Springer Nature remains neutral with regard to jurisdictional claims in published maps and institutional affiliations.

\section{Authors and Affiliations}

\section{Tom St Quinton ${ }^{1} \cdot$ Ben Morris $^{1} \cdot$ Dylan Pickering $^{2} \cdot$ Debbie M. Smith ${ }^{3}$}

Tom St Quinton PhD

t.stquinton@leedstrinity.ac.uk

1 School of Social and Health Sciences, Leeds Trinity University, Brownberrie Lane, LS18 5HD Leeds, UK

2 Gambling Treatment and Research Clinic, School of Psychology, University of Sydney, Sydney, Australia

3 Manchester Centre for Health Psychology, School of Health Sciences, The University of Manchester, Manchester, UK 